\title{
A drink containing amino acids and chromium picolinate improves postprandial glycemia at breakfast in healthy, overweight subjects
}

\author{
Elin Ostman, Anna Forslund, Rickard Oste and Inger Bjorck
}

Food for Health Science Centre, Lund University, P.O. Box 124, SE-221 00 Lund, Sweden

Corresponding author: Elin Ostman, Food for Health Science Centre, Lund University, P.O. Box 124, SE-221 00 Lund, Sweden

Submission Date: September 22, 2016, Acceptance date: February 20, 2016: Publication date: February 28, 2016

Citation: Ostman E., Forslund A., Oste R., Bjorck I. A drink containing amino acids and chromium picolinate improves postprandial glycemia at breakfast in healthy, overweight subjects. Functional Foods in Health and Disease 2017; 7(2): 88-97

\section{ABSTRACT \\ Background: Chromium (Cr) and certain amino acids (AA) have been individually shown to improve postprandial glycemia.}

Method: The present randomized, controlled, cross-over trial in 19 healthy, overweight subjects (age $51 \pm 1 \mathrm{y}$ and BMI $27.3 \pm 0.3 \mathrm{~kg} / \mathrm{m}^{2}$; mean $\pm \mathrm{SEM}$ ) evaluated a combination of leucine, isoleucine, valine, lysine, and threonine (5AA) with Cr. Postprandial glycemia and insulinemia were measured following a bread meal, served with carbonated water (Ref) or carbonated water containing 5AA, Cr-picolinate ( $\mathrm{CrPic})$ or a combination $(5 \mathrm{AA}+\mathrm{CrPic})$.

Results: The 5AA+CrPic and 5AA respectively lowered the incremental glucose peak $(\mathrm{P}<$ 0.001 ) by almost $30 \%$ compared to Ref. No significant differences in incremental insulin peaks were found. However, during the first 15 minutes 5AA induced a higher insulin response $(+112 \%$; $\mathrm{p}<0.01)$ compared to Ref. Interestingly, 5AA+CrPic reduced the initial AA-induced insulin increase by more than $50 \%$, indicating improved insulin economy.

Conclusions: These observations suggest that a drink containing both 5AA and $\mathrm{CrPic}$ attenuate postprandial glycemia in healthy "at risk" subjects.

Keywords: Amino acids, chromium picolinate, postprandial glycemia, insulin economy, drink

\section{INTRODUCTION}

Low glycemic diets are associated with reduced inflammatory tonus (CRP, adiponectin) [1], lowered oxidative stress $(\mathrm{NF} \kappa \beta)$ [2] and reduced risk of type 2 diabetes mellitus (T2DM), and heart disease [3]. Ingestion of whey proteins has been reported to positively affect glucose metabolism and increase satiety [4]. One suggested mechanism is the insulinogenic properties 
of whey protein, and particularly the hypoglycemic effect, which has been related to five specific amino acids (5AA; leucine, isoleucine, valine, threonine, and lysine) that rise rapidly in blood upon whey ingestion [5]. Accordingly, the efficiency of the 5AA to reduce the postprandial glycemic response has been repeatedly proven in healthy subjects [5, 6]. Furthermore, the specific mix of 5AA has been shown to improve hepatic steatosis and glucose tolerance in mice [7]. In another setting, a mix of AA containing leucine, isoleucine, valine, cysteine, and methionine improved postprandial glucose responses in healthy overweight subjects [8]. When it comes to the role of AA in diabetes, Solerte et al, has shown that supplementation of the diet with all essential AA, significantly improved glucose regulation (lowered HbA1c) in poorly controlled T2DM subjects [9].

Another dietary factor of interest in relation to glycemic regulation is chromium [10]. However, most of the studies made on Cr supplementation have been performed in T2DM subjects, where improved effects on glycemic control, improved lipid profiles, attenuated weight gain, and reduced central fat distribution have been reported [11-14]. In hyperglycemic subjects, dietary supplementation with $\mathrm{Cr}$ decreased glucose and insulin responses to an oral glucose challenge [15]. Moreover, in subjects at high risk of developing T2DM, increased insulin sensitivity was reported in the absence of effects on body composition after 8 months intake of Cr-picolinate (CrPic) [16]. However, there are also trials in non-diabetic or T2DM subjects which have failed to show metabolic improvements associated with $\mathrm{Cr}$ [17-20].

We hypothesized that a combination of 5AA and $\mathrm{Cr}$ in a drink could synergistically improve postprandial glycemia when co-ingested with a composite and challenging high glycemic sandwich breakfast in healthy, overweight subjects. Three carbonated test drinks were studied containing either the $5 \mathrm{AA}, \mathrm{CrPic}$ or a combination of both $(5 \mathrm{AA}+\mathrm{CrPic})$. Carbonated water without the addition of either ingredient was used as reference. The test and reference drinks, respectively, were provided with a sandwich meal consisting of white wheat bread, butter, and marmalade, and postprandial blood glucose and insulin were registered for 3 hours. Additionally, subjective ratings of appetite sensations were registered.

\section{METHODS}

\section{Study design}

The study was a randomized, controlled, cross-over, single blind human trial. Nineteen subjects were included (11M:8F). The volunteers were non-smoking, aged $51 \pm 1 \mathrm{y}$ (mean \pm SEM), considered to be healthy, and had body mass indices in the overweight range (BMI $27.3 \pm 0.3 \mathrm{~kg} / \mathrm{m}^{2}$, mean $\pm \mathrm{SEM}$ ). Five persons were receiving either of the following medication: one blood-thinning (Trombyl), two hypotensive (Enalapril and Tenormin, respectively), one vitamin B supplementation (Behepan), and one allergen extract from grass pollen (Grasax). These medications were not considered to affect the parameters tested in this study and were therefore allowed. However, the volunteers were asked to not take the medication in the morning before the test. All subjects participated as volunteers, gave their written informed consent, and were aware of the possibility of withdrawing from the study without explanation at any time. The study was approved by the Regional Ethical Board at Lund University (Dnr 2010/499).

The test and reference breakfast meals were served in random order over four separate occasions after an overnight fast, approximately one week apart. The subjects were instructed to eat the same type of dinner in the evening before each test day and a late evening snack consisting of white wheat bread with spread and a drink. Furthermore, they were asked to not 
perform any extensive exercise, drink alcohol, or eat fiber-rich food on the day before each visit. All meals were well tolerated and subjects finished them within 10-15 minutes as requested.

\section{Reference and test drinks}

Five AA (L-leucine, L-isoleucine, L-threonine, L-valine, and L-lysine) and CrPic were added either alone or in combination to the three test drinks. Both $\mathrm{CrPic}$ and the 5AA were kindly provided by Einar Willumsen (Brondby, Denmark). Reference and test drinks were named as follows: water with grapefruit aroma and no other ingredients (Ref), water with grapefruit aroma and 5AA (5AA), water with grapefruit aroma and $\mathrm{CrPic}(\mathrm{CrPic})$, and water with grapefruit aroma and both 5AA and $\mathrm{CrPic}(5 \mathrm{AA}+\mathrm{CrPic})$. CrPic was added in the amount of $500 \mu \mathrm{g}$, which corresponds to $62 \mu \mathrm{g}$ of $\mathrm{Cr}$. The molar ratio of AA was 1.1:1.1:1.7:2.1:2.2 for Val:Ile:Lys:Thr:Leu. Carbon dioxide and aroma was added to improve palatability. No sugar was added to the drinks. More details on the drinks are presented in Table 1. The carbonated drinks were prepared the day before each test (except for Ref that was provided by the flavoring company in one batch).

Table 1. Ingredients in the test and reference drinks.

\begin{tabular}{llllll}
\hline & $\begin{array}{l}\text { Water } \\
(\mathrm{g})\end{array}$ & $\begin{array}{l}\text { Amino } \\
\operatorname{acids}^{1}(\mathrm{~g})\end{array}$ & CrPic $(\mu \mathrm{g})$ & Aroma $(\mathrm{g})$ & Total weight $^{3}(\mathrm{~g})$ \\
\hline Ref & 312.0 & - & - & $\mathrm{X}^{2}$ & 312.0 \\
CrPic & 308.0 & - & 500 & 0.19 & 312.0 \\
5AA & 305.1 & 6.9 & - & 0.19 & 312.0 \\
5AA+CrPic & 301.1 & 6.9 & 500 & 0.19 & 312.0 \\
\hline
\end{tabular}

CrPic, Chromium picolinate; ${ }^{1}$ Molar ratio of the amino acids; 1.1:1.1:1.7:2.1:2.2 for Val:Ile:Lys:Thr:Leu, respectively; ${ }^{2}$ Aroma added by flavoring company in unknown amount; ${ }^{3}$ Total weight without aroma taken in account.

\section{Breakfast meal}

A standardized, composite breakfast meal was served along with each of the reference or test drinks. The breakfast meal consisted of white wheat bread (WWB) (Dollarfranska, Lockarp, Sweden) corresponding to $50 \mathrm{~g}$ available starch [21] with $24 \mathrm{~g}$ orange marmalade (Onos, Orkla Foods, Sweden) and $10 \mathrm{~g}$ butter (Bregott, Arla Foods, Sweden). All breakfast meals contained the same amount of carbohydrates and fat, whereas protein varied depending on whether or not AA were included in the accompanying drink. The nutritional composition of the sandwich meal was $64.0 \mathrm{~g}$ carbohydrates, $9.1 \mathrm{~g}$ protein, and $11.7 \mathrm{~g}$ fat with an energy content of $380.1 \mathrm{kcal}$. The AA in the drinks (5AA and 5AA+CrPic) corresponded to $6.9 \mathrm{~g}$ protein equaling $27.6 \mathrm{kcal}$. Hence, the meals with the AA-containing drinks contained 407.8 $\mathrm{kcal}$ and a total of $16.0 \mathrm{~g}$ protein.

\section{Blood analysis}

Capillary blood samples were taken at time 0 (fasting) and at 15, 30, 45, 60, 90, 120, and 180 minutes after breakfast to determine glucose and insulin. Blood glucose was evaluated directly using a B-glucose analyzer (HemoCue Glucose $201^{+}$Analyser, HemoCue AB, Angelholm, Sweden). Serum was separated by centrifuging samples for 5 min (3000 rpm, 
Eppendorf mini spin, F-45-12-11) and frozen at $-18^{\circ} \mathrm{C}$ until analysis. Insulin was measured using an enzyme immunoassay kit (Mercodia AB, Uppsala, Sweden) on an integrated immunoassay analyzer (CODA Open Microplate System; Bio-Rad Laboratories, Hercules, CA, USA).

\section{Subjective appetite rating}

During each session, the test subjects were asked to rank their subjective appetite at $0,15,30$, $45,60,90,120$, and 180 minutes on a $100 \mathrm{~mm}$ visual analogue scale (VAS) ranging from "not at all" to "extremely". Feeling of satiety, feeling of hunger, and desire to eat were evaluated.

\section{Calculations and statistical analysis}

Nineteen subjects participated in the study, with all but one completing all test meals. Of the nineteen participants, one subject was excluded from final analysis as he was indicated as a statistical outlier with Grubbs' test for 5 time points (Minitab Statistical Software, version 16, State College, PA). As a result, data from 18 subjects was further analyzed. One person's data is missing for 5AA due to illness $(n=17)$. One person missed VAS scoring for the subjective appetite ratings; therefore, one set of appetite scores are missing for the $5 \mathrm{AA}+\mathrm{CrPic}$ drink $(\mathrm{n}=17)$.

All results are expressed as mean \pm SEM. The incremental area under the curve (iAUC) for glucose and insulin was calculated according to the trapezoidal method, excluding areas below fasting level. Total areas under the curve (tAUC) were calculated for feeling of satiety, feeling of hunger, and desire to eat respectively, using GraphPad Prism (ver 5, GraphPad Software, San Diego, CA, USA). Incremental peaks (iPeaks) were calculated per individual for glucose and insulin respectively, as the maximum elevation from baseline. The glucose profile (GP) was calculated by dividing the duration (the time the curve remained above baseline) by the iPeak [22].

All statistical calculations were performed in Minitab Statistical Software (versions 14 and 16, State College, PA, USA). To evaluate significances for iAUCs of glucose and insulin, in addition to iPeaks, GI, II, and GP, analysis of variance (ANOVA) was used followed using Tukey's pair wise comparisons test. For appetite ratings, analysis of variance was conducted with covariate (ANCOVA) using total area values and the 0 -values as covariate. In the cases where the residuals were not normally distributed, Box Cox transformation was applied before analysis with ANOVA and ANCOVA.

Time $\times$ treatment interactions were analyzed for glucose and insulin responses using a mixed model (PROC MIXED in SAS, release 8, SAS Institute Inc., Cary, NC) with repeated measures and an autoregressive covariance structure. The subjects were the random variable and the fasting values the covariate.

\section{RESULTS}

\section{Postprandial glycemia}

The glycemic responses are represented in Figure 1 and Table 2. A main effect for product $(\mathrm{P}=0.0346)$ and a product $\times$ time interaction was found $(\mathrm{P}=0.0020) .5 \mathrm{AA}+\mathrm{CrPic}$ and 5AA respectively resulted in significantly lower glucose response (iAUC) compared to Ref during the first hour. The iPeak $(\Delta \mathrm{mmol} / \mathrm{L})$ was significantly lower for 5AA and 5AA+CrPic compared to both Ref and CrPic respectively. GP was significantly higher after 5AA+CrPic 
and 5AA compared to Ref. There was no significant difference in glycemia between CrPic and Ref at any time.

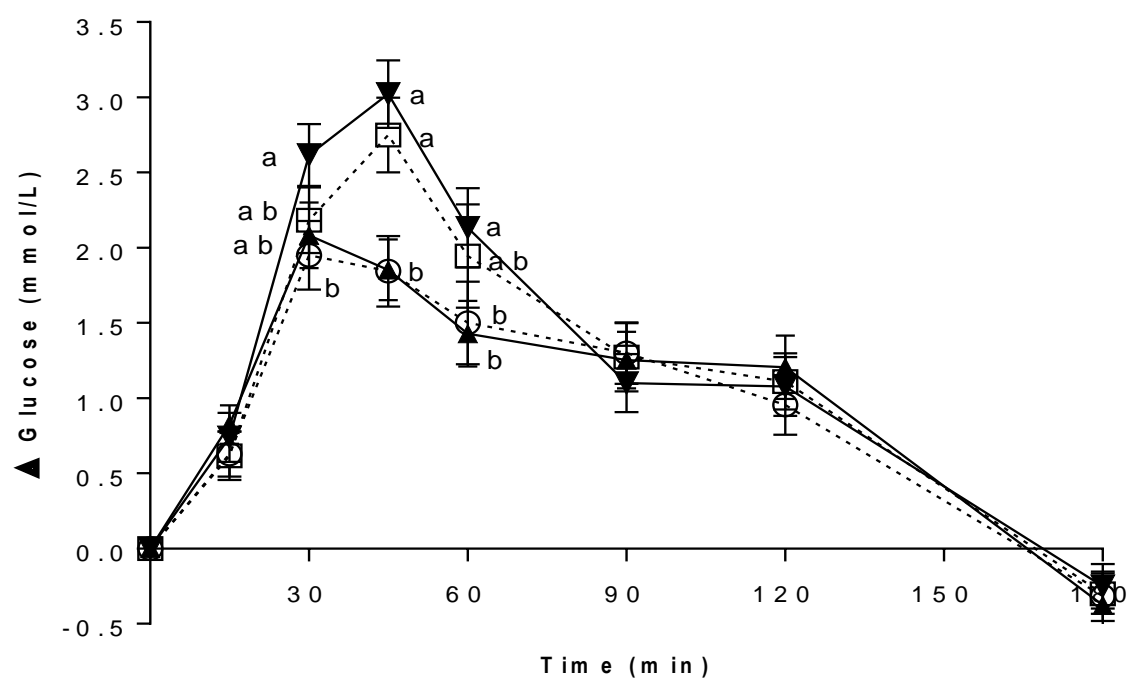

Fig. 1 Glycemic responses following intake of a breakfast with four different test drinks: reference (Ref) ( $\mathbf{\nabla}$ ), chromium picolinate (CrPic) ( $\square$ ), mix of amino acids leucine, isoleucine, valine, lysine and threonine $(5 \mathrm{AA})(\boldsymbol{\Delta})$ and $5 \mathrm{AA}+\mathrm{CrPic}(\mathrm{O})$. Values expressed as mean $\pm \mathrm{SEM} ; \mathrm{n}=18$ (except for 5AA, $\mathrm{n}=17$ ). Incremental glucose values at each time point, not sharing the same letter are significantly different.

Table 2. Glucose and insulin responses after intake of a standardized breakfast with different test and reference drinks.

\begin{tabular}{|c|c|c|c|c|}
\hline & Ref & CrPic & $5 \mathrm{AA}$ & $5 \mathrm{AA}+\mathrm{CrPic}$ \\
\hline \multicolumn{5}{|l|}{ Glucose } \\
\hline$i A U C^{1} 0-60 \min (\Delta \%)^{2}$ & $111.7 \pm 8.0 a(-)$ & $98.1 \pm 9.5 a b(-12)$ & $82.1 \pm 7.4 b(-26)$ & $78.1 \pm 8.4 b(-30)$ \\
\hline$i A U C^{1} 0-120 \min (\Delta \%)^{2}$ & $192.8 \pm 17.3(-)$ & $182.2 \pm 20.7(-5)$ & $159.5 \pm 16.2(-17)$ & $155.0 \pm 18.1(-20)$ \\
\hline$i A U C^{1} 0-180 \min (\Delta \%)^{2}$ & $223.9 \pm 22.7(-)$ & $212.6 \pm 25.0(-5)$ & $195.5 \pm 21.9(-13)$ & $183.5 \pm 22.5(-18)$ \\
\hline iPeak, mmol/L $(\Delta \%)^{2}$ & $3.3 \pm 0.2 a(-)$ & $3.0 \pm 0.2 a(-10)$ & $2.3 \pm 0.2 b(-29)$ & $2.4 \pm 0.2 b(-27)$ \\
\hline $\mathrm{GP}^{3}, \min \cdot(\mathrm{mM})^{-1}(\Delta \%)^{2}$ & $52 \pm 3 a(-)$ & $60 \pm 5 a b(+15)$ & $74 \pm 5 b(+42)$ & $75 \pm 7 b(+44)$ \\
\hline \multicolumn{5}{|l|}{ Insulin } \\
\hline$i A U C^{1} 0-15 \min (\Delta \%)^{2}$ & $0.41 \pm 0.12 a(-)$ & $0.40 \pm 0.07 a(-3)$ & $0.87 \pm 0.16 b(+112)$ & $0.61 \pm 0.14 a b(+49)$ \\
\hline$i A U C^{1} 0-60 \min (\triangle \%)^{2}$ & $7.52 \pm 0.66 a(-)$ & $7.17 \pm 0.84 a(-5)$ & $10.9 \pm 1.11 b(+45)$ & $9.31 \pm 1.06 a b(+24)$ \\
\hline$i A U C^{1} 0-120 \min (\Delta \%)^{2}$ & $14.2 \pm 1.2 a(-)$ & $14.1 \pm 1.4 a(-1)$ & $20.6 \pm 2.3 b(+45)$ & $19.0 \pm 2.3 b(+34)$ \\
\hline$i A U C^{1} 0-180 \min (\Delta \%)^{2}$ & $16.6 \pm 1.7 a(-)$ & $16.6 \pm 1.9 a(-)$ & $23.9 \pm 3.1 b(+44)$ & $21.8 \pm 2.7 b(+31)$ \\
\hline iPeak, nmol/L $(\Delta \%)^{2}$ & $0.24 \pm 0.02 a b(-)$ & $0.24 \pm 0.02 b(+3)$ & $0.30 \pm 0.03 a(+22)$ & $0.29 \pm 0.03 a b(+17)$ \\
\hline
\end{tabular}

Ref, Reference; CrPic, Chromium picolinate; 5AA, 5 amino acids (Val:Ile:Lys:Thr:Leu); iAUC, incremental area under the curve; iPeak, incremental peak; GP, glycemic profile; ${ }^{1}$ Expressed as min•mmol/L; ${ }^{2}$ Percentage change from Ref.; ${ }^{3}$ Residuals not normally distributed after Box Cox transformation. Values are expressed as mean \pm SEM; $n=18$ (except for 5AA, $n=17$ ). Values on the same row not sharing the same letter are significantly different $(\mathrm{p}<0.05)$. 


\section{Postprandial insulinemia}

The insulin responses are represented in Figure 2 and Table 2. A main effect was found for the product $(\mathrm{p}=0.0035)$, as well as a product $\times$ time interaction $(\mathrm{p}<0.0001)$. During the first 15 minutes after start of breakfast, the 5AA induced a significant increase in insulin compared to Ref. The insulin increase during the same time for 5AA+CrPic was about half of the 5AA response, and did not differ significantly from Ref or CrPic. The overall insulin responses (iAUC 0-180 $\mathrm{min}$ ) were significantly higher for both 5AA and 5AA+CrPic compared to Ref and CrPic. The iPeak ( $\Delta \mathrm{mmol} / \mathrm{L})$ was significantly higher after 5AA compared with CrPic but not different from Ref or 5AA+CrPic.

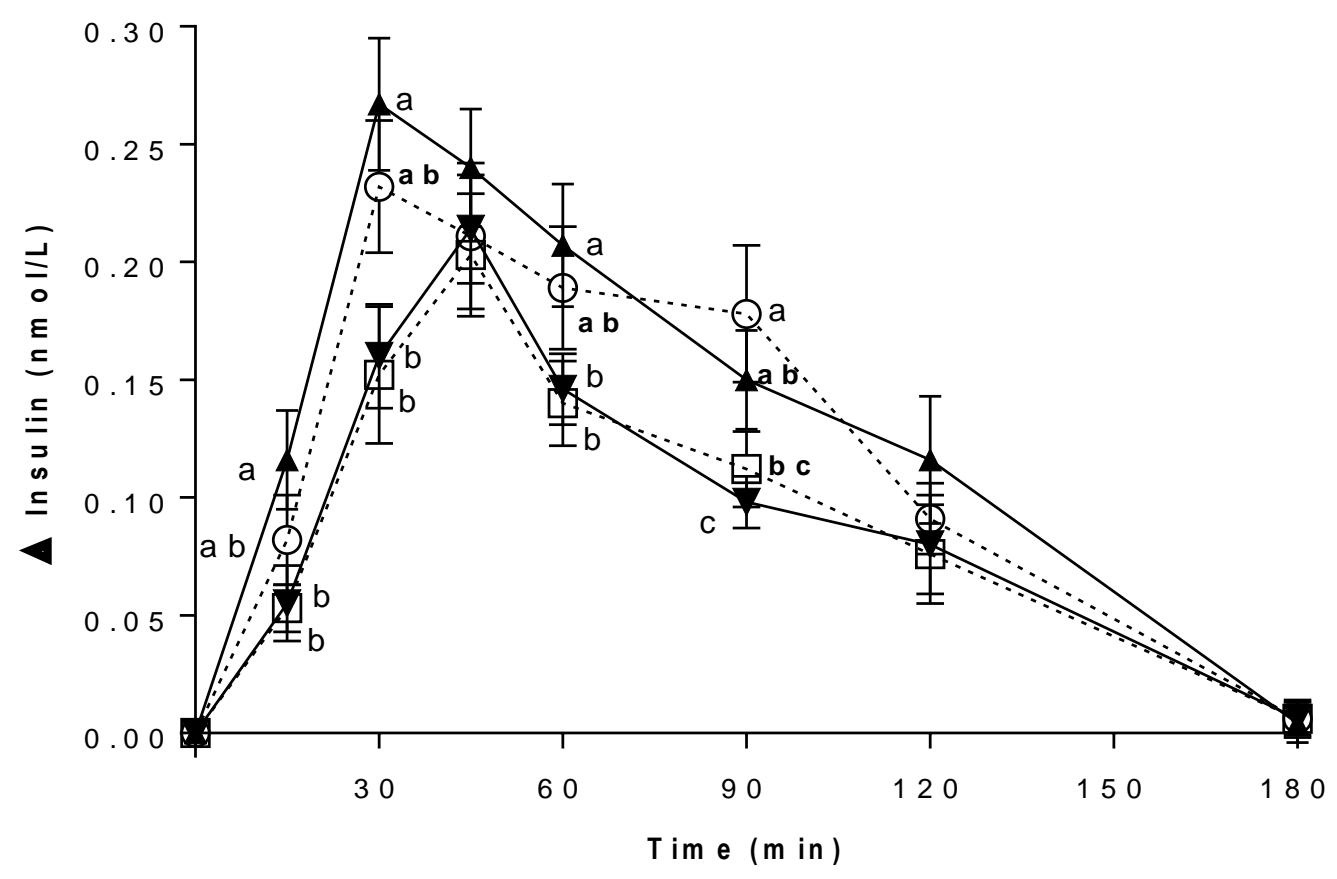

Fig. 2 Insulinemic responses following intake of a breakfast with four different test drinks: reference (Ref) ( $\boldsymbol{\nabla})$, chromium picolinate (CrPic) ( $\square$ ), mix of amino acids leucine, isoleucine, valine, lysine and threonine (5AA) ( $\mathbf{A})$ and 5AA+CrPic (O). Values expressed as mean \pm SEM; $\mathrm{n}=18$ (except for 5AA, $\mathrm{n}=17$ ). Incremental insulin values at each time point, not sharing the same letter are significantly different.

\section{Subjective appetite ratings}

Among the subjective appetite ratings there was one significant difference observed for feeling of satiety (tAUC 0-180), with a lower rating for CrPic compared to 5AA (results not shown).

\section{DISCUSSION}

This study demonstrates that both AA-containing test drinks (5AA and 5AA+CrPic respectively) caused similar improvements in glycemia after breakfast, as measured by lowered iAUC 0-60 min, reduced iPeak and increased GP, compared to the Ref drink. However, a difference between 5AA+CrPic and 5AA was found in the insulin response. Whereas a significant early increase in postprandial plasma insulin was discovered following the 5AA meal (+112\%, iAUC 0-15 min) compared to Ref, the corresponding insulin increase after the meal with $5 \mathrm{AA}+\mathrm{CrPic}(+49 \%)$ was about half and not different from Ref. The 
finding that the specific AA have an insulin stimulating and glucose reducing effect is in line with previous results $[5,6]$. However, there seems to be less insulin needed to obtain the same glucose lowering effect if $\mathrm{CrPic}$ is added to the 5AA-drink. Therefore, the drink with $5 \mathrm{AA}+\mathrm{CrPic}$ appeared to improve insulin economy.

The fact that the peak of the mean glucose responses occur at $30 \mathrm{~min}$ for 5AA and $5 \mathrm{AA}+\mathrm{CrPic}$ and at $45 \mathrm{~min}$ for Ref and $\mathrm{CrPic}$ indicate that the early insulin release caused by 5AA is effective in counteracting the glucose rise after intake of a challenging breakfast. In fact, the improved first phase insulin responses have been reported as important for overall glucose regulation and decreased risk of T2DM [23]. Furthermore, in a previous study the early insulin release (0-30 $\mathrm{min}$ ) to a carbohydrate challenge supplemented with whey protein and 5AA was correlated to the beneficial impact of glycemia, as manifested by lowered glucose responses and increased glycemic profile in healthy, normal weight subjects [6]. In the present study, no such correlations were found (results not presented); additionally, it should be noted that in the study by Gunnerud, et al. [6] the 5AA was taken as a pre-meal drink, while in the present study the 5AA were ingested together with the breakfast meal.

The present results suggest that the addition of CrPic to the AA-containing drink blunted the insulin responses elicited by the 5AA. To our knowledge, such an acute insulin saving effect of Cr-supplementation has not been reported before. In previous acute and longer term studies of Cr-supplementation, beneficial effects on glycemic control have been reported as reduced acute glucose responses and glycemic peaks, as well as reduced fasting glucose and improved insulin sensitivity $[12,24]$. Furthermore, $\mathrm{Cr}$ has been hypothesized to be insulin sensitizing both in cell [25] and animal [26] studies. Acute effects of CrPic have been reported for doses ranging from 200-1000 $\mu \mathrm{g}[12,15,24]$. In this study, the dose was set to $500 \mu \mathrm{g} \mathrm{CrPic}$ and no significant glucose lowering effect was found. However, results regarding the efficacy of dietary supplementation with $\mathrm{Cr}$ on indicators of metabolism are inconclusive [27]. One reason for this could be that the acute effect of $\mathrm{Cr}$ may differ in between subjects [24]. Differences in responses between individuals may originate from differences in $\mathrm{Cr}$ status where a deficiency [15] or a state of insulin resistance may influence the metabolic response to Cr-supplementation [28]. Furthermore, the number of responders and non-responders to $\mathrm{Cr}$ was investigated in the present study. A responder was defined as a subject showing more than $5 \%$ decrease in postprandial glucose iAUC (0-180 min) compared to the corresponding meal with the Ref drink. Nine out of the 18 subjects were defined as responders to $\mathrm{Cr}$, with this number being considered too low to proceed with any statistical evaluation. It should also be noted that Cefalu et al [29] proposed that the division of subjects into responders or non-responders based on glucose responses may conceal the ones benefiting from $\mathrm{Cr}$ on insulin sensitivity, which thereby falsely characterizes them as non-responders. It should be noted that the potential insulin sensitizing effects of $\mathrm{Cr}$ in the present study may have been masked by the insulinogenic effects of the AA. Furthermore, most studies have been conducted over a longer time period, and it cannot be excluded that a longer-term intervention could have revealed differences between the 5AA and 5AA+CrPic drinks.

Both whey proteins and $\mathrm{Cr}$ have been suggested to modulate appetite and attenuate body weight gain [12, 30, 31]. In this study, hunger, satiety, and the desire to eat were measured but no differences were found between any of the meals. It should be noted though that the study was not powered to detect differences in appetite ratings. 


\section{CONCLUSIONS}

Drinks containing 5AA (with and without $\mathrm{CrPic}$ ) attenuated postprandial glycemia to a challenging sandwich breakfast by $25-30 \%$. An early insulin increase seems to be of importance for the benefits on postprandial glycemic regulation and a combination of 5AA with $\mathrm{CrPic}$ improved insulin economy. Therefore, it is concluded that the 5AA+CrPic drink, when co-ingested with a high glycemic breakfast meal, affected the glycemic response in healthy "at risk" subjects beneficially, and thereby may circumvent pro-oxidative and/or proinflammatory conditions associated with oscillatory glycemic episodes as described in acute and longer term studies.

\section{Abbreviations}

CrPic, Chromium picolinate; 5AA, 5 amino acids (leucine, isoleucine, valine, lysine and threonine); Ref, Reference meal; iAUC, incremental area under the curve; iPeak, incremental peak; GP, glycemic profile

\section{Competing interests}

Elin Ostman and Inger Bjorck jointly own a patent application describing the combination of protein and mineral that has been studied. Double Good AB holds a license to the IPR. Rickard Oste is a co-inventor of the patent application and adjunct professor at Food for Health Science Centre, at part-time from his employment at Aventure AB. Anna Forslund declares no conflict of interests.

\section{Authors' contributions}

E. Ostman, R. Oste, and I. Bjorck designed the study; AF performed the practical part of the study, in addition to the statistical analysis of the data with supervision from EO; EO and AF drafted the manuscript and all authors engaged in the manuscript work. EO had primary responsibility for the final content. All authors have read and approved the final manuscript.

\section{Acknowledgements and funding}

This study was supported by the Lund University Antidiabetic Food Center, a VINNOVA VINN Excellence Center (Grant number: 2008/8).

\section{REFERENCES}

1. Neuhouser, M.L., et al., A low-glycemic load diet reduces serum C-reactive protein and modestly increases adiponectin in overweight and obese adults. J Nutr, 2012. 142(2): p. 369-74.

2. Dickinson, S., et al., High-glycemic index carbohydrate increases nuclear factorkappaB activation in mononuclear cells of young, lean healthy subjects. Am J Clin Nutr, 2008. 87(5): p. 1188-93.

3. Barclay, A.W., et al., Glycemic index, glycemic load, and chronic disease risk - a meta-analysis of observational studies. American Journal of Clinical Nutrition, 2008. 87(3): p. 627-37.

4. Sousa, G.T., et al., Dietary whey protein lessens several risk factors for metabolic diseases: a review. Lipids Health Dis, 2012. 11: p. 67.

5. Nilsson, M., J.J. Holst, and I.M. Björck, Metabolic effects of amino acid mixtures and whey protein in healthy subjects: studies using glucose-equivalent drinks. American Journal of Clinical Nutrition, 2007. 85(4): p. 996-1004. 
6. Gunnerud, U.J., et al., Effects of pre-meal drinks with protein and amino acids on glycemic and metabolic responses at a subsequent composite meal. PLoS One, 2012. 7(9): p. e44731.

7. Noguchi, Y., et al., Ketogenic essential amino acids modulate lipid synthetic pathways and prevent hepatic steatosis in mice. PLoS One, 2010. 5(8): p. e12057.

8. Wang, B., et al., Amino acid mixture acutely improves the glucose tolerance of healthy overweight adults. Nutr Res, 2012. 32(1): p. 30-8.

9. Solerte, S.B., et al., Improvement of blood glucose control and insulin sensitivity during a long-term (60 weeks) randomized study with amino acid dietary supplements in elderly subjects with type 2 diabetes mellitus. American Journal of Cardiology, 2008. 101(11A): p. 82E-88E.

10. Cefalu, W.T. and F.B. Hu, Role of Chromium in Human Health and Diabetes. Diabetes Care, 2004. 27(11): p. 2741-2751.

11. Suksomboon, N., N. Poolsup, and A. Yuwanakorn, Systematic review and metaanalysis of the efficacy and safety of chromium supplementation in diabetes. Journal of Clinical Pharmacy and Therapeutics, 2014. 39(3): p. 292-306.

12. Martin, J., et al., Chromium picolinate supplementation attenuates body weight gain and increases insulin sensitivity in subjects with type 2 diabetes. Diabetes Care, 2006. 29(8): p. 1826-32.

13. Albarracin, C.A., et al., Chromium picolinate and biotin combination improves glucose metabolism in treated, uncontrolled overweight to obese patients with type 2 diabetes. Diabetes Metab Res Rev, 2008. 24(1): p. 41-51.

14. Sharma, S., et al., Beneficial effect of chromium supplementation on glucose, HbAlC and lipid variables in individuals with newly onset type-2 diabetes. $\mathrm{J}$ Trace Elem Med Biol, 2011. 25.

15. Anderson, R.A., et al., Supplemental-chromium effects on glucose, insulin, glucagon, and urinary chromium losses in subjects consuming controlled low-chromium diets. Am J Clin Nutr, 1991. 54(5): p. 909-16.

16. Cefalu, W.T., et al., Effect of chromium picolinate on insulin sensitivity in vivo. Journal of Trace Elements in Experimental Medicine, 1999. 12(2): p. 71-83.

17. Gunton, J.E., et al., Chromium supplementation does not improve glucose tolerance, insulin sensitivity, or lipid profile: a randomized, placebo-controlled, double-blind trial of supplementation in subjects with impaired glucose tolerance. Diabetes Care, 2005. 28(3): p. 712-713.

18. Kleefstra, N., et al., Chromium Treatment Has No Effect in Patients With Poorly Controlled, Insulin-Treated Type 2 Diabetes in an Obese Western Population. Diabetes Care, 2006. 29(3): p. 521.

19. Kleefstra, N., et al., Chromium treatment has no effect in patients with type 2 diabetes in a Western population: a randomized, double-blind, placebo-controlled trial. Diabetes Care, 2007. 30(5): p. 1092-6.

20. Iqbal, N., et al., Chromium picolinate does not improve key features of metabolic syndrome in obese nondiabetic adults. Metab Syndr Relat Disord, 2009. 7(2): p. 14350.

21. Holm, J., et al., A rapid method for the analysis of starch. Starch-Starke, 1986. 38(7): p. 224-226.

22. Rosén, L.A., et al., Endosperm and whole grain rye breads are characterized by low post-prandial insulin response and a beneficial blood glucose profile. Nutr J, 2009. 8: p. 42.

23. Del Prato, S. and A. Tiengo, The importance of first-phase insulin secretion: implications for the therapy of type 2 diabetes mellitus. Diabetes Metab Res Rev, 2001. 17(3): p. 164-74. 
24. Frauchiger, M.T., C. Wenk, and P.C. Colombani, Effects of acute chromium supplementation on postprandial metabolism in healthy young men. J Am Coll Nutr, 2004. 23(4): p. 351-7.

25. Chen, G., et al., Chromium activates glucose transporter 4 trafficking and enhances insulin-stimulated glucose transport in 3T3-L1 adipocytes via a cholesterol-dependent mechanism. Mol Endocrinol, 2006. 20(4): p. 857-70.

26. Chen, W.Y., et al., Chromium supplementation enhances insulin signalling in skeletal muscle of obese KK/HlJ diabetic mice. Diabetes Obes Metab, 2009. 11(4): p. 293303.

27. Balk, E.M., et al., Effect of chromium supplementation on glucose metabolism and lipids: a systematic review of randomized controlled trials. Diabetes Care, 2007. 30(8): p. 2154-63.

28. Wang, Z.Q., et al., Phenotype of subjects with type 2 diabetes mellitus may determine clinical response to chromium supplementation. Metabolism, 2007. 56(12): p. 1652-5.

29. Cefalu, W.T., et al., Characterization of the metabolic and physiologic response to chromium supplementation in subjects with type 2 diabetes mellitus. Metabolism, 2010. 59(5): p. 755-62.

30. Anton, S.D., et al., Effects of chromium picolinate on food intake and satiety. Diabetes Technol Ther, 2008. 10(5): p. 405-12.

31. Veldhorst, M.A., et al., Dose-dependent satiating effect of whey relative to casein or soy. Physiol Behav, 2009. 96(4-5): p. 675-82. 